\title{
PRENATAL MANAGEMENT WITH DIGOXIN AND SOTALOL COMBINATION FOR FETAL SUPRAVENTRICULAR TACHYCARDIA: CASE REPORT AND REVIEW OF LITERATURE
}

VANITA SURI, ANISH KEEPANASERIL, NEELAM AGGARWAL, RAJESH VIJAYVERGIYA ${ }^{1}$

\section{ABSTRACT}

Sustained fetal supraventricular tachycardia (SVT) with a heart rate of approximately 210 bpm may lead to increased atrial and venous pressures and congestive heart failure. There is no clear consensus regarding the best drug-treatment regimens for fetal SVT. However, considerable nonrandomized experience in the transmaternal treatment of fetal SVT is available with a number of antiarrhythmic agents. We report a case of fetal supraventricular tachyarrhythmia with hydrops detected at 32 weeks that was managed with combination of oral digoxin and sotalol and review management guidelines available in the literature.

Key words: Digoxin, fetal supraventricular tachyarrhythmia, hydrops, sotalol DOI: 10.4103/0019-5359.56117

\section{INTRODUCTION}

Sustained tachyarrhythmias are the most clinically relevant rhythm disturbances in the fetus. Sustained fetal supraventricular tachycardia (SVT) with a heart rate of approximately $210 \mathrm{bpm}$ may lead to congestive heart failure..$^{[1,2]}$ There is no clear consensus regarding the best drug-treatment regimens for fetal SVT. Digoxin has been recommended as first-line therapy in cases of SVT with cardiac failure, but recent evidence suggests that its transplacental passage may be impaired

Departments of Obstetrics and Gynecology and Cardiology, Postgraduate Institute of Medical

Education and Research, Chandigarh, India

Correspondence:

Dr. Vanita Suri,

Department of Obstetrics and Gynecology,

Postgraduate Institute of Medical Education and Research,

Sector 12, Chandigarh, India.

E-mail: dr_vanita_suri@yahoo.co.in in the presence of hydrops. ${ }^{[2,3]}$ Other agents such as flecainide and sotalol have been tried as first-line agents but with adverse events. ${ }^{[3,4]}$ We report a case of fetal supraventricular tachyarrhythmia with hydrops detected at 32 weeks and managed with combination of oral digoxin and sotalol.

\section{CASE REPORT}

A 32-year-old multiparous lady was referred to our antenatal clinic with fetal hydrops with tachycardia detected during a routine scan. Antenatal period was apparently uncomplicated till 32 weeks, when the ultrasound showed hydrops fetalis and fetal tachycardia. There was no history of fever, intake of any drugs or thyrotoxicosis. In her first pregnancy, she underwent cesarean section for nonprogress of labor with fetal distress at term; gave birth to a girl child, who is 6 years old without any cardiovascular complications. She had 2 
late-first-trimester abortions subsequently. On admission, the fetal heart rate was $250 \mathrm{bpm}$ .Fetal echo showed a structurally normal heart and the fetal heart rate to be $223 \mathrm{bpm}$ making a diagnosis of supraventricular tachycardia, which was found to be of incessant nature [Figure 1]. She was started on digoxin 0.25 $\mathrm{mg}$ OD and sotalol $40 \mathrm{mg} \mathrm{BD}$ at 32 weeks and was monitored with serum electrolytes and electrocardiography (QTc prolongation). Three days later, fetal heart rate was found to be $250 \mathrm{bpm}$, and the sotalol dose was increased to $60 \mathrm{mg}$ BD. Subsequently the fetal heart rate decreased within 12 days of initiation of therapy and remained stabilized in the range of 140 to $150 \mathrm{bpm}$. Fetal surveillance was done with serial ultrasonography, cardiotocography and fetal echocardiography. Hydrops disappeared within 5 days of stabilization of fetal heart rate at $150 \mathrm{bpm}$. The fetal heart rate remained stable at 120 to $140 \mathrm{bpm}$ till delivery [Figure 2]. Her autoimmune workup and TORCH screening were negative. Results of thyroid function test were normal. She underwent elective cesarean section at 37 weeks for previous cesarean section with oligohydramnios. She delivered a male child with APGAR score of 8.9 , weighing $2.68 \mathrm{~kg}$. The baby had a heart rate of 120 to $170 \mathrm{bpm}$. Neonatal ECG showed a regular rhythm with no evidence of pre-excitation. Neonatal echo showed structurally normal heart with no episodes of SVT. The baby was discharged on day 7 of his life. On follow-up at 1 year of age, the child is having normal sinus rhythm with no recurrence of tachycardia.

\section{DISCUSSION}

Supraventricular tachycardia (SVT) occurs at an unknown frequency prenatally,

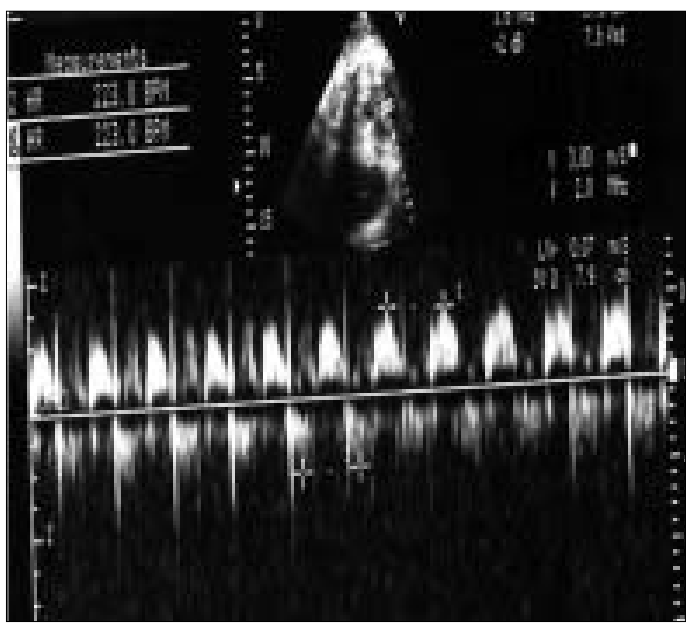

Figure 1: 2-D fetal $\mathrm{ECHO}$ showing fetal heart rate before treatment

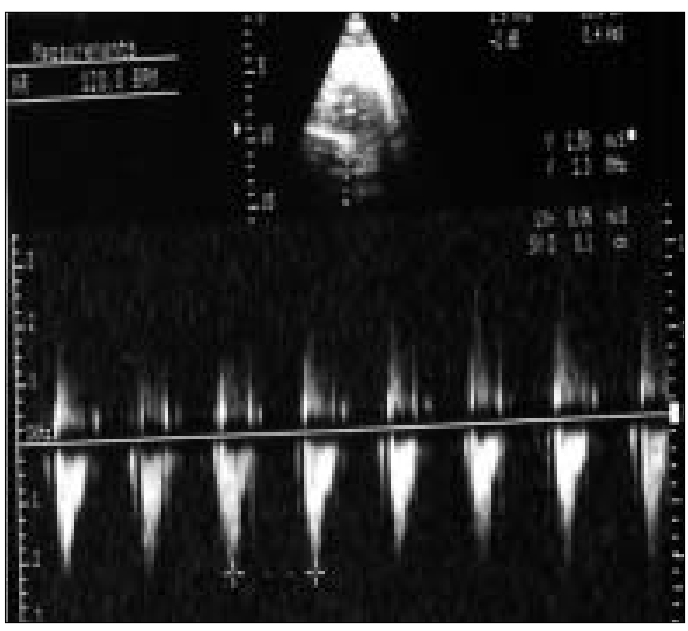

Figure 2: 2-D fetal ECHO showing fetal heart rate after treatment

approximately 1 in 500-1000 pregnancies. ${ }^{[3]}$ It can be due to different mechanisms, most commonly due to $\mathrm{AV}$ re-entrant tachycardia. ${ }^{[1,3]}$

Auscultation can be used as a screening method to detect tachycardia. Cardiotocography may be used for continuous fetal monitoring during initiation of transplacental therapy and during labor. Using 
the M-mode or Doppler in ultrasound, the fetal cardiac rhythm can be assessed by determining the relationship between atrial and ventricular activations. ${ }^{[2]}$ Using fetal echocardiogram, a detailed assessment for concomitant structural heart disease, myocardial function and the degree of hemodynamic compromise can be done. ${ }^{[3]}$ In the index case, as there was a regular mitral inflow velocity followed by aortic outflow velocity on pulse wave Doppler, the atrial flutter or fibrillation with variable $\mathrm{AV}$ conduction was excluded. However, the exact mechanism of supraventricular tachycardia in the fetus was difficult to decide with Doppler. Magnetocardiogram (MCG) and Doppler myocardial deformation analysis are the newer methods used for the diagnosis. ${ }^{[1-3,5]}$

There is a large spectrum of severity in SVT, ranging from incidentally detected episode to fetal hydrops and intrauterine death, and the contributing factors to cardiac failure are unclear. Guntheroth et al. reported no cases of fetal hydrops when the rate of SVT was less than 230 bpm. ${ }^{[5]}$ Other authors have found no relationship between rate or duration of SVT and risk of fetal hydrops, which has been reported even in the presence of intermittent SVT. ${ }^{[6,7]}$ Other complications include polyhydramnios, preterm labor and abruptio placentae.

Spontaneous resolution of SVT has been reported to occur in utero, during delivery or in the neonatal period. ${ }^{\left[{ }^{[8]}\right.}$ When SVT is intermittent with no signs of cardiac failure, patients are followed with weekly echocardiographic examination. More intensive monitoring is required (i.e., twice weekly or every other day) when SVT is sustained. Evidence of hemodynamic compromise (e.g., hydrops fetalis) is an indication for hospitalization. ${ }^{[6-8]}$

In the term fetus, urgent delivery and direct therapy are provided to the newborn. In the nonhydropic term fetus, vaginal delivery can be considered. ${ }^{[2,3]}$ If the fetus presents with intermittent episodes of tachycardia with no hemodynamic compromise, close monitoring can be considered; but if it is sustained tachyarrhythmia, then rapid and permanent conversion to sinus rhythm to prevent or resolve congestive heart failure is needed. ${ }^{[2]}$

There are no proper guidelines regarding the start of therapy and the mode of application, so management of SVT should be tailored to the individual case. ${ }^{[2]}$ Transplacental drug transfer decreases as a result of this placental edema in the presence of hydrops. ${ }^{[2,5]}$ The choice of treatment is controversial as there are no controlled data available about the drug therapy. ${ }^{[2,3,5]}$ Digoxin is still considered as the drug of choice in treating fetal SVT, with a single-drug efficacy greater than $50 \%$ and having a positive inotropic action, while all other agents are neutral or negative inotropes. ${ }^{[9]}$ Use of Flecainide or procainamide as a first-line agent may be limited due to its higher pro-arrhythmic activity and its fetal-negative inotropic actions, which may accelerate the development of hydrops. ${ }^{[9]}$ Sotalol, a beta adrenergic antagonist, has been recently introduced in the treatment of SVT in fetuses with and without fetal hydrops. ${ }^{[4]}$ Potential pro-arrhythmic effects of sotalol remain a source of concern, which excludes it as the drug of first choice in uncomplicated SVT. In cases with severe hydrops and poor cardiac function, due to its possible negative 
inotropic effect, the drug should always be administered in association with digoxin.

After successful anti-arrhythmic treatment, dosage is maintained until delivery with weekly controls of the fetal cardiac rhythm. Cesarean section is not indicated due to the rhythm disorder only, if the heart rate is controlled. ${ }^{[2,3,10]}$ One third or more of patients with SVT prenatally will be free of further SVT, presumably due to involution of the abnormal pathway. Newborns are treated (usually with a b-blocker) for at least 6 to 12 months if the SVT 1) spontaneously recurs early after birth or (2) is easily inducible during transesophageal electrophysiological study. ${ }^{[3]}$

Recent studies show a high mortality rate, viz., $27 \%$ to $50 \%$, without treatment, whereas a fetal mortality of merely $5 \%$ to $10 \%$ was reported when anti-arrhythmic therapy was administered..$^{[1,2]}$ Fetal hydrops is commonly considered to be a negative prognosticator. In our case, hydrops resolved 5 days after normalization of the fetal heart rate and approximately 3 weeks after the initiation of therapy, and the neonatal period was uneventful. The baby is currently under follow-up in the cardiology clinic without any complications at 1 year of age.

In conclusion, combination therapy with digoxin and sotalol can be used as the first line of management in fetuses presenting with SVT in the late trimester. As combination therapy requires lower dose, it reduces the risk of the pro-arrhythmic effects and other side effects of both the drugs. Oral digoxin therapy also avoids the need of therapeutic drug-level monitoring, which is required with intravenous therapy to monitor for the side effects.

\section{REFERENCES}

1. Strasburger JF, Cheulkar B, Wichman HJ. Perinatal arrhythmias: Diagnosis and management. Clin Perinatol 2007;34:627-52.

2. Strasburger JF. Fetal arrhythmias. Progr Pediatr Cardiol 2000;11:1-17.

3. Jaeggi ET, Nii M. Fetal brady- and tachyarrhythmias: New and accepted diagnostic and treatment methods. Semin Fetal Neonatal Med 2005;10:504-14.

4. Sonesson SE, Fouron JC, Wesslen-Eriksson E, Jaeggi E, Winberg P. Foetal supraventricular tachycardia treated with sotalol. Acta Paediatr 1998;87:584-7.

5. Perles Z, Gavri S, Rein AJTT. Tachyarrhythmias in the fetus: State of the art diagnosis and treatment. Progress in Pediatric Cardiology 2006: 22; 95107.

6. Simpson JM, Sharland GK. Fetal tachycardias: Management and outcome of 127 consecutive cases. Heart 1998;79:576-81.

7. Simpson LL, Marx GR, D'Alton ME. Supraventricular tachycardia in the fetus: Conservative management in the absence of hemodynamic compromise. J Ultrasound Med 1997;16:459-64.

8. Cuneo BF, Strasburger JF. Management strategy for fetal tachycardia. Obstet Gynecol 2000;96:575-81.

9. Allan LD, Chita SK, Sharland GK, Maxwell D, Priestley K. Flecainide in the treatment of fetal tachycardias. Br Heart J 1991;65:46-8.

10. Guntheroth WG, Cyr DR, Shields LE, Nghiem HV. Rate-based management of fetal supraventricular tachycardia. J Ultrasound Med 1996;15:453-8.

Source of Support: Nil. Conflict of Interest: None declared. 